\title{
E28 A METHOD FOR STATIC-BASED UP-GRIDDING
}

Rami M. Younis and Jef Caers Stanford University, Petroleum Engineering, Stanford, CA 94305-2220, USA

\begin{abstract}
A method for up-gridding geostatistical reservoir models is proposed. The method generates well-adapted coarse-grid grids for a given realization and target coarse-scale dimensions. While most current grid generation methods are based on either local or global dynamic (flow rate dependent) measures, in this paper, we show that the inclusion of both local and global static information, can greatly improve computational cost while maintaining accurate flow response. Static property information is analyzed to infer a measure of spatially variability in terms of the relative influence of connected bodies on reservoir flow response. These bodies consist of wellconnected permeability falling within a range of permeability values. Bodies may have a strong impact on local and global flow behavior; knowledge of the relative location of such bodies in a given realization may render an accurate coarse-scale representation of the fine scale models in terms of flow response. An investigation of the method under a set of realistic synthetic $2 \mathrm{D}$ and $3 \mathrm{D}$ reservoirs is conducted. Sensitivity to strength of geological continuity, and to flow boundary conditions are considered for various scale-up ratios. The results indicate accurate reproductions of the flow simulation response when compared to that of the fine scale realizations. A comparative analysis of the method with well-established static methods is also conducted.
\end{abstract}

\section{Introduction}

Numerous existing grid selection methods rely on dynamic responses, such as streamline simulations, to identify the locations of the fine cells through which flow tends to frequent $[4,5$, 6 , and 8]. Such dynamic-based up-scaling methods have proven to be effective in accurately preserving the flow response of the fine-scale in cases where boundary conditions remain relatively constant. However, in practice, dynamic information is generally not constant throughout the life of a reservoir, and as a result, such dynamic-based methods tend to lack robustness. Moreover, they remain computationally tedious for the scale of modern geostatistical models. On the other hand, static-based methods relying on the permeability heterogeneity itself as a measure for designing up-scaled grids, and in turn, may be more robust.

Several authors have developed such static methods [1, 2, 3, and 7]. Of those methods, the ones that are applicable to any type of geological model approach the problem of up-gridding in one of two ways. The geobody approach defines regions of similar and spatially connected permeability in such a way that each region can be treated as a single coarse block with a single effective permeability. The local variance minimization approach attempts to minimize the variance of permeability over a variational function, minimizing block variance. In effect, the local variance minimization approach amounts to contrast-seeking solutions that avoid constructing coarse-grid blocks containing sharp fine-scale permeability contrast. 
The objective of this discussion is to propose a novel geobody-based static up-gridding method that is general to any geology, target coarse-grid topology, and dimensionality. The properties of the method are investigated, and compared to those of another well-established method; the local variance minimization approach of the Garcia et al [1] Elastic Grid Adjustment method (DEGA).

\section{Qualitative arguments for the Geobody approach}

The geobody approach is centered around the hypothesis that regions of relatively similar and spatially connected permeability can have a strong impact on flow response. The presence or absence of geobody structures such as channels is not the only important factor affecting flow. In order to summarize what features of geobodies may be particularly important in impacting flow response, four attributes of geobodies key to our proposed method are identified: the degree of continuity and size, relative predominance of continuous permeability, interface permeability transition and contrast, and anisotropy and geometry.

The proposed method aims to design coarse grids of target dimensions $\left\{n c_{x}, n c_{y}, n c_{z}\right\}$ for any given fine-scale grid of dimensions $\left\{n_{x}, n_{y}, n_{z}\right\}$ such that the bodies most important to flow response are preserved. To achieve this, firstly a formal framework for defining permeability bodies is required such that any realization can be de-composed into a map of bodies. Our approach will be developed for Cartesian grids but can be extended to other types, including unstructured grids.

\section{Defining bodies}

Bodies need to be defined such that they capture the main features of a reservoir's geology, with particular emphasis on the attributes that affect flow the most. At the same time, bodies need to be as homogeneous as possible in order to make the calculation of a body's effective permeability more accurate. These criteria are satisfied by defining bodies to enclose permeability nodes that are spatially continuous, and are within a subset of the range of permeability observed in the whole field.

The range of permeability in a realization $l$ can be spanned by a set of permeability intervals $\Delta^{(l)}{ }_{i}, i=1, \ldots, N_{i}$. We consider the interval spacing is quantile-based, i.e. each interval has the same frequency of permeability values. Hence, an important problem is in the choice of an appropriate $N_{t}$. For each interval, all nodes occupied by permeability values within this interval form a set $S_{i}$. For any two nodes in $S_{i}$, they are said to be connected in $S_{i}$ if there exists a path between them consisting entirely of nodes in $S_{i}$. A body is a subset of nodes in $S_{i}$ that are all connected to each other.

Thus for each interval $\Delta^{(l)}{ }_{i}, i=1, \ldots, N_{t}$ there is one $S_{i}$, and there will be at least one body for each $S_{i} \neq \varnothing, i=1, \ldots, N_{t}$. All such bodies belong to their interval uniquely since the intervals themselves do not overlap by definition, and each node in the realization can only belong to a single body. The process of finding nodal networks to form bodies is repeated over all $N_{t}$ intervals. The result of this process is a set of $N_{b}$ connected regions (bodies).

This process of de-composing a realization is illustrated with an example. Figure la shows a 2Dchannel realization $l$. In order to define bodies on this realization, first, a set of 4 permeability intervals is determined as shown in Figure $1 \mathrm{~b}$. For each interval, a pseudo-realization can be 
composed, representing the corresponding sets of nodes $S_{i}$-- Figure 2. For each pseudorealization in Figure 2, connected bodies are found, and assigned a unique code (integers)-Figure 3. By superimposing all of these integer-labeled bodies, Figure 4 shows the channel realization de-composed into a map of bodies. In total, $N_{b}=225$ bodies exist in this realization based on the hypothetical choice of the $\Delta^{(l)}{ }_{i}, i=1, \ldots, 4$ intervals.

Permeability intervals $\Delta^{(l)}{ }_{i}, i=1, \ldots, N_{t}$

The definition of bodies relies on the definition of permeability intervals $\Delta^{(l)}{ }_{i}, i=1, \ldots, N_{t}$. The importance of the number of intervals $N_{t}$ can be understood by considering that for very coarse target up-gridding resolution, bodies should be generated at a larger scale such that the major flow units may be discerned, at the less dramatic expense of lumping fine-scale detail. On the other hand, at higher up-scaling resolution targets, it would then be more 'affordable' to add more emphasis to important sub-regions within important flow units.

The number of intervals determines two important opposing properties of bodies; namely the total number of bodies obtained $N_{b}$, and the range of permeability within each body. Both factors are considered in selecting the $N_{t}$ suitable for a given target set of coarse dimensions $\left\{n c_{x}, n c_{y}, n c_{z}\right\}$. Ultimately, it is optimal if $N_{t}^{*}$ intervals would result in $N_{b}^{*}=n c_{x} . n c_{y} . n c_{z}$ since in this idealistic case, no selection of bodies may be necessary, i.e. all bodies become single coarse grid-blocks. This number of intervals corresponding to the optimal scenario is not known a priori, and is searched for iteratively.

Practical bounds for an iterative search are defined at two extreme scenarios. At one extreme, a single permeability interval will result in one body. Consequently, a lower acceptable limit $N_{t}^{\text {low }}=2$ is set. On the other extreme, it would be unrealistically ideal if on setting $N_{t}=n c_{x} \cdot n c_{y} \cdot n c_{z}, N_{b}=n c_{x} \cdot n c_{y} . n c_{z}$ bodies are obtained, where each body belongs exclusively to a single permeability interval. Consequently, an upper limit of $N_{t}^{h i g h}=n c_{x} . n c_{y} . n c_{z}$ is set. Almost any constrained ID numerical optimization algorithm can be applied to search for $N_{t}^{*}$. In the current implementation, a simple gradient search algorithm was implemented.

\section{Characterizing body attributes}

In order to relate bodies to actual grids, they must first be geometrically parameterized. Furthermore, it is necessary to quantify the four most important attributes of bodies that can indicate their level of influence on flow response. Bodies have two types of properties that summarize the set of nodes they contain. The first type of property is a geometric description, and the second type of property consists of scalar values: the number of nodes in a body (size), and the arithmetic or any other type of average of body-node permeability.

More specifically, the geometric properties are a parameterization of a body's external surface. The topological form of this parameterization should match the topology of the required target coarse-grid. The implementation of the method in this discussion is for non-uniform Cartesian grids, and for that reason, the surfaces of all bodies are to be approximated by rectangular bounding boxes. The projections of bounding surfaces are termed body borders - Figure 5 a. Such surface parameterizations can only be approximate since the topology of bodies in a realization is not restricted to conform to that of a regular grid i.e. rectangular. 
In order to grid a body more accurately than by using a single set of body-borders, we introduce additional grid-lines, termed neighboring borders-- Figure $5 \mathrm{~b}$. The combination of body-borders and neighboring-borders allows more accurate modeling of the transition between a body and the surrounding rock.

With these scalar and geometric properties, the challenge of designing a coarse grid is in selecting bodies and their borders. In order to propose an algorithm to select bodies and borders, we develop a set of heuristic functions, which can relate bodies and their properties to the importance of preserving them in a coarse grid.

\section{Heuristically quantifying a bodies impact on flow response}

In order to rank bodies according to their impact on flow, we consider two scales of flow behavior. At one scale, broadly categorized permeability regions, e.g. shale and sandstone, impact flow response through their internal properties, i.e. effective permeability, size, and geometry. At a smaller scale, nodal permeability transitions between interfaces also impact flow response through transitional property gradients. Hypothetically, large scale permeability regions can be associated with the global direction and magnitude of flow, whereas the nodal scale features are most associated with how, where, and when local cross flows may occur. We define the term influence as the effect that a particular body feature such as size, has on flow response, and an influence function as a quantifiable measure of influence associated with a body feature. A body-scale influence function and a nodal-scale influence function are defined to quantify the level of influence of bodies and their borders on either scale. Based on a coupling of these two factors, we will define a grid-line selection algorithm that selects the most influential borders in terms of their impact on flow.

\section{Body-scale influence functions}

Heterogeneity in permeability fields may be regarded as variation around a characteristic dominant permeability. With this outlook, bodies with similar permeability values occupying the largest volumes in a realization may be considered as dominant. Less dominant bodies are regarded as anomalies, which can be identified and isolated from the dominant 'background' to capture heterogeneity. In the case of body-scale influence, the body size and difference in body permeability from the dominant permeability are important factors.

For the set of all bodies $b_{k}, k=1, \ldots, N_{b}$ comprising a realization $l$, each body has size $n_{k}$, defined as the number of grid nodes incorporated in the body. The dominant bodies are taken to be the subset of all bodies $b_{j}$ belonging to the single $\Delta^{(l)}{ }_{i}$ that has the bodies with the largest size on the average. This single interval is denoted as $\Delta^{(l)} d o m$. The body-scale influence function $I_{k}{ }^{\text {bod }}, k=1, \ldots, N_{b}$ is defined for each body in a realization as the deviation of the body's average permeability from that of the average body permeability in $\Delta^{(l)}$ dom , as well as on the size of the body in question $n_{k}, k=1, \ldots, N_{b}$. For a given body $\beta$, denoting the average permeability in $\Delta_{d o m}^{(l)}$ as $k_{d o m}^{(l)}$, the body's size as $n_{\beta}$, and the arithmetic averaged permeability of the body $\beta$ as $\bar{k}_{\beta}^{(l)}$, the body's body-scale influence $I_{\beta}{ }^{\text {bod }}$ is defined as follows:

$$
I_{\beta}^{\text {bod }}=n_{\beta} \frac{\left(\bar{k}_{\beta}^{(l)}-k_{d o m}^{(l)}\right)^{2}}{k_{d o m}^{(l)}}
$$


A body's influence is proportional to its size and to the deviation of its average permeability from the dominant permeability squared, and has units of permeability.

\section{Nodal-scale influence functions}

The requirement for nodal-scale accuracy at interfaces between adjacent influential bodies may be evaluated by quantifying transitions in permeability about body-borders and neighbors. This measure can be defined as the ratio of the average node-pair permeability difference along a border to the measure of variation between the average permeability of all nodes on both sides of a border and the realization's dominant permeability.

This determination of the nodal-influence term is presented here for a hypothetical body-border $x_{b}$ with neighboring grid-line $x_{b+1}=x_{b}+1$. The nodal permeability along these two grid-lines are $k^{(l)}\left(x_{b}, i, j\right), i=1, \ldots, n_{y}, j=1, \ldots, n_{z}$ and $k^{(l)}\left(x_{b}, i, j\right), i=1, \ldots, n_{y}, j=1, \ldots, n_{z}$ respectively. The nodal-scale influence function for border $x_{b}$ is defined as:

$$
I_{b}^{\text {nod }}=\frac{\sum_{j=1}^{n_{x}} \sum_{i=1}^{n_{y}}\left|k^{(l)}\left(x_{b}, i, j\right)-k^{(l)}\left(x_{b+1}, i, j\right)\right|}{\left|\bar{k}_{b}^{(l)}-k_{d o m}^{(l)}\right|},
$$

Where $k_{d o m}^{(l)}$ is the realization's dominant permeability, and the average permeability local to nodes along the border $\bar{k}_{b}^{(l)}$ is defined as:

$$
\bar{k}_{b}^{(l)}=\frac{\sum_{j=1}^{n_{z}} \sum_{i=1}^{n_{y}} k^{(l)}\left(x_{b}, i, j\right)+k^{(l)}\left(x_{b+1}, i, j\right)}{2 n_{y} n_{z}}
$$

Combining the two influence functions in selection

For all $N_{b}$ bodies, and $m$ body-borders and neighbors in a realization $l$, we have defined a set of body-scale influence terms $I_{k}^{b o d}, k=1, \ldots, N_{b}$ and a set of local nodal-scale influence terms $I_{i}^{\text {nod }}, i=1, \ldots, m$ respectively. To select body-borders as non-uniform grid-lines on the basis of

$I_{k}^{b o d}$, and $I_{i}^{\text {nod }}$, both factors need to be coupled to achieve a balance between body-scale, and nodal-scale heterogeneity. The selection algorithm aims to integrate the idea of balancing body and nodal-scale accuracy by weighing whether it would be more beneficial to select a body with a low body influence, or to add neighboring borders around a body with higher body influence.

The minimum number of bodies to be captured is one. Therefore the body-borders of the body with highest body-scale influence are always selected. After that point, there is a choice between emphasizing this body further by selecting more neighboring borders around it, or alternatively, selecting the next most important body. Such decisions are based on the relative magnitude of $I^{\text {bod }}$ and $I^{\text {nod }}$. The algorithm terminates when the target number of coarse gridlines is selected.

\section{Overview of a benchmark method}

In order to investigate the effectiveness of the proposed method, the Elastic Grid Adjustment method developed by Garcia et al [1] was used as an alternative method for comparison. The Elastic Grid Adjustment (DEGA) method is a well-established static method that minimizes a 
measure of coarse-block heterogeneity variationaly in order to design coarse-grids. The measure of heterogeneity is a function of a block's internal variance, and its volume. The crux of the method is in considering a coarse grid as a system of springs connecting a set of sliding nodes by elastic grid-lines. The challenge of minimizing internal block property variation is thus reduced to assigning elasticity or spring constants to grid-lines.

\section{Comparative case studies}

The two up-gridding methods discussed were applied to a set of realizations generated by the GSLIB program sgsim. Effective permeability was evaluated by the single-phase pressure solver flowsim over the output coarse grids from both methods. These up-scaled realizations were then input to the finite difference reservoir simulator ECLIPSE to obtain the flow responses.

\section{Two-dimensional realizations}

Twenty realizations were generated, conditioned to data sets from three well locations, and containing $100 \times 1 \times 100=10,000$ nodes. The realizations were generated using spherical variograms with ranges chosen such that there was considerable zonal anisotropy, with varying lateral continuity. The permeability fields were transformed log-normally with a similar mean and variance of approximately 5 and 1 respectively. For each of these realizations, 4 target coarse grid dimensions were set. One such case is discussed here for a non-uniform target grid resolutions of $20 \times 1 \times 10=200$ blocks.

Figure 6 and Figure 7 show the gridding results for the Geobody, and DEGA methods respectively. Figure 8 shows the two flow-response target profiles for both methods compared to the fine realization flow responses. It is apparent from these figures that DEGA and Geobody generated grids demonstrate accurate reproduction of the target flow response. Moreover, both methods seem to pick out the major high permeability layers in the realization, rendering visually comparable coarse models. Computational time to generate coarse grids with the Geobody implementation is on the order of fractions of a second for these realizations on a Pentium 866 $\mathrm{MHz}$ desktop.

\section{Multiple producers in $3 D$ realizations}

Ten $3 \mathrm{D}$ realizations were generated containing $50 \times 50 \times 100=250,000$ nodes in an otherwise similar fashion as the previous $2 \mathrm{D}$ case set. Target coarsening dimensions were chosen to reduce the number of blocks by at least one to two orders of magnitude. The reservoirs occupied a physical volume of $1000 \times 1000 \times 500$ feet. A single, regular five-spot production pattern was used to produce the models at constant bottom hole pressure (BHP) for 250 days.

Figure 9 shows the up-scaling results for the Geobody, and DEGA methods applied to one such realization at coarse dimensions of $20 \times 20 \times 10$ blocks. Both methods capture high permeability layers in the reservoir. The Geobody grids are qualitatively less uniform than the DEGA grids, particularly at the coarse up-scaling dimensions. Figure 10 shows the water cut target profile results for both methods compared to the fine realization flow response. It is apparent from this figure that the accuracy of reproduction of the target flow response is consistent with the results discussed previously.

\section{Conclusions}

Both methods demonstrate that coarse grid design on the basis of static information can result in accurate reproduction of flow response. This was demonstrated for substantially low coarse scale dimensions up to a reduction of model size by two orders of magnitude. Both methods 
qualitatively consume little CPU time-on the order of seconds for fine realizations with 250,000 cells.

\section{References}

[1] M.H. Garcia, A.G. Journel, and K. Aziz, "An automatic grid generation and adjustment method for modeling reservoir heterogeneities," SPE \#21471, May 1992.

[2] R.M. Younis and J. Caers, "A method for reservoir scale-up by static-based non-uniform gridding," Stanford Center for Reservoir Forecasting (SCRF) 2001 Annual Report, May 2001.

[3] D. Li, A.S., and L.W. Lake, "Global scale-up of reservoir model permeability with local grid refinement," Journal of Petroleum Science and Engineering, Vol. 14, pp. 1-13, 1995.

[4] S. Verma and K. Aziz, "Two and three dimensional flexible grids for reservoir simulation," Proceedings of the $5^{\text {th }}$ European Conference on the Mathematics of Oil Recovery, Leoben 1996.

[5] L.J. Durlofsky, R.C. Jones, and W.J. Milliken, "A non-uniform coarsening approach for the scale up of displacement processes in heterogeneous porous media," Advances in Water Resources, 1997.

[6] T.C. Wallstrom, S. Hou, M.A. Christie, and L.J. Durlofsky, " Application of a new two-phase upscaling technique to realistic reservoir cross sections," SPE \#51939, 1999.

[7] D. Li and B.Beckner, "Optimal uplayering for scaleup of multimillion-cell geologic models," SPE \#62927, 2000.

[8] A. Castellini, M.G. Edwards, and L.J. Durlofsky, "Flow based modules for grid generation in two and three dimensions," Proceedings of the $7^{\text {th }}$ European Conference on the Mathematics of Oil Recovery, 2000.

(a)

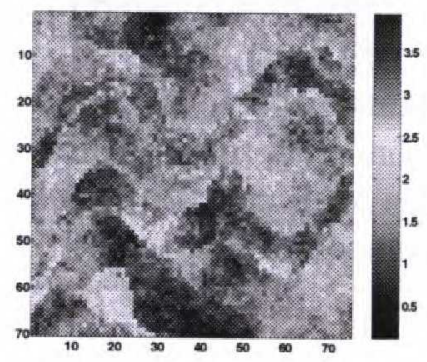

(b)

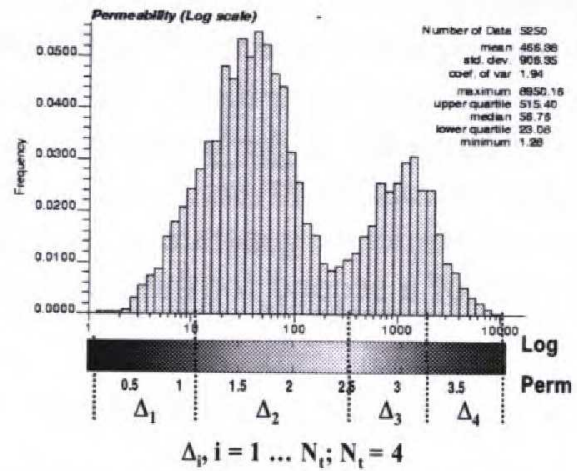

Figure 1: Permeability intervals for a channel realization; (a) Realization, and (b) permeability intervals.

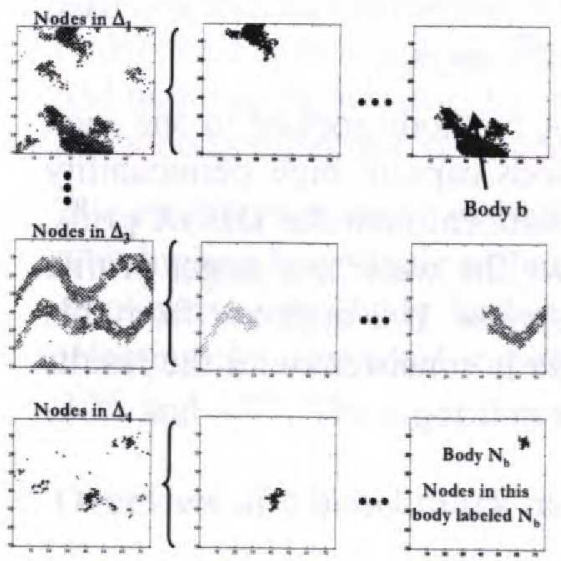

Figure 3: Connected sets of nodes in single pseudorealizations form bodies.

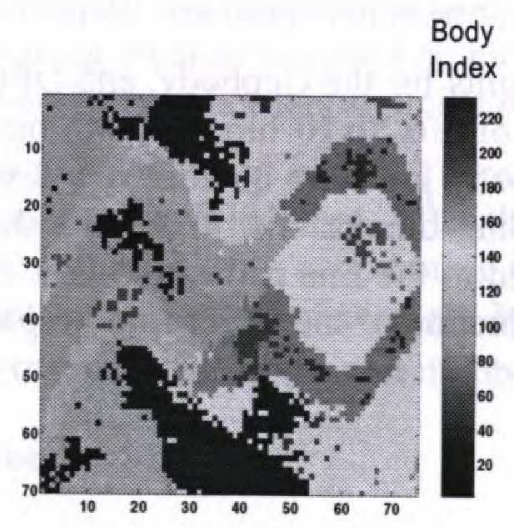

Figure 4: Body index map.

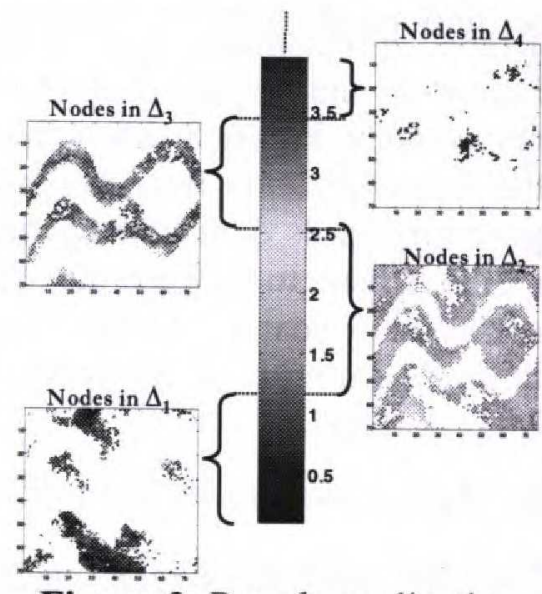

Figure 2: Pseudo-realizations obtained for each interval.

(a)

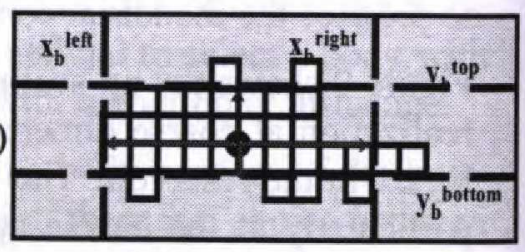

(b)

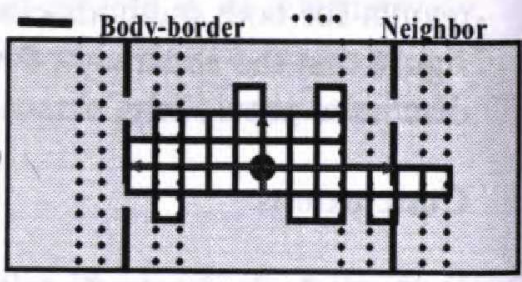

Figure 5: (a) Body-borders, and (b) Neighbors 
(a)

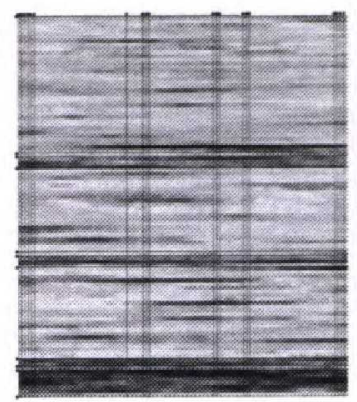

(b)

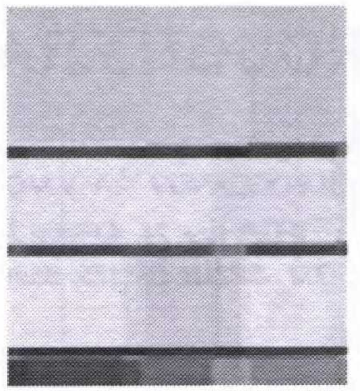

Figure 6: Geobody grid results (a) Coarse-grid overlay, and (b) Corresponding up-scaled model. (a)

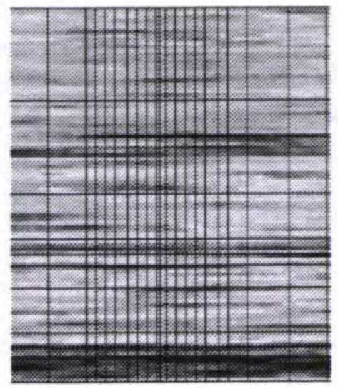

(b)

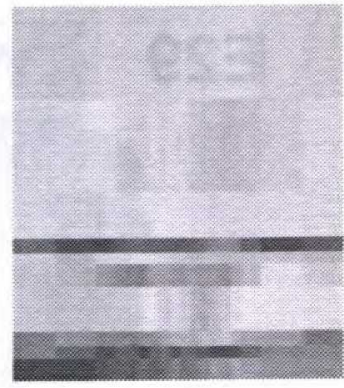

Figure 7: DEGA grid results (a) Coarse-grid overlay, and (b) Corresponding up-scaled model.

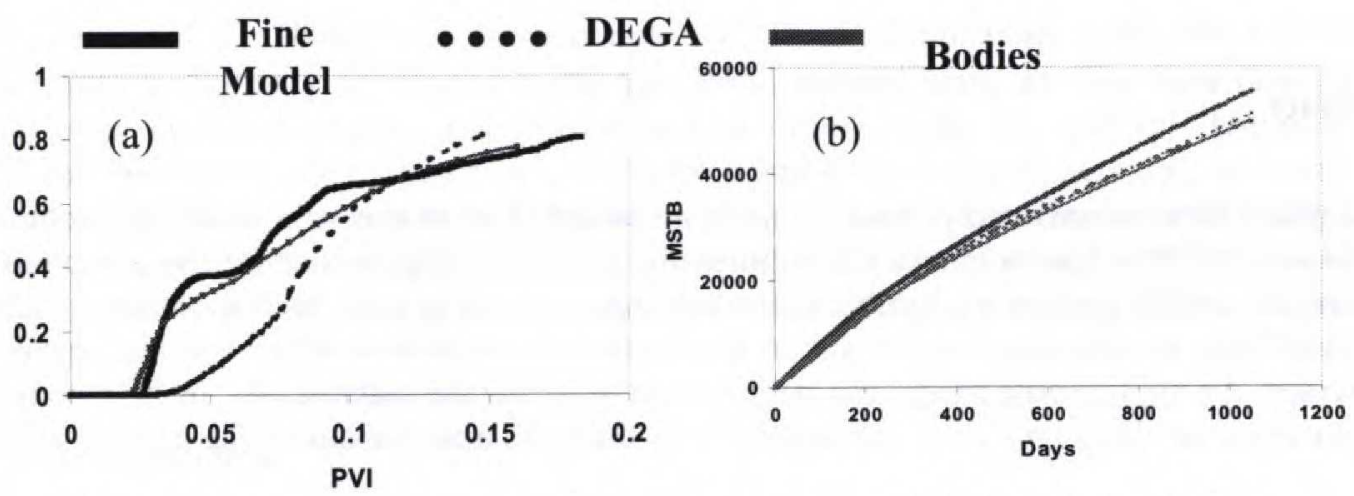

Figure 8: Target response results (a) Water cut, and (b) Total cumulative

(a)

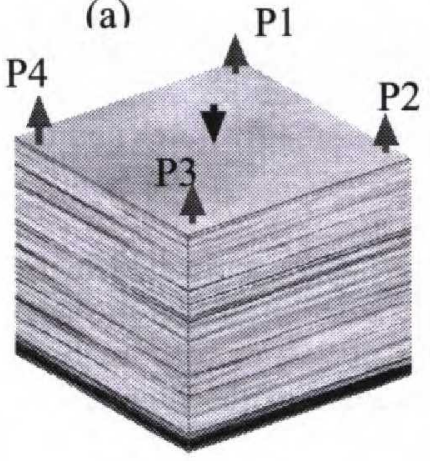

(b)

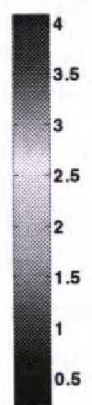

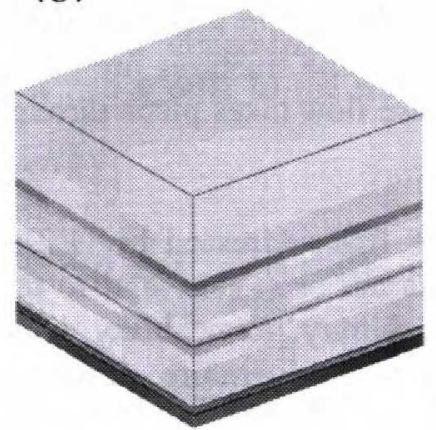

(c)

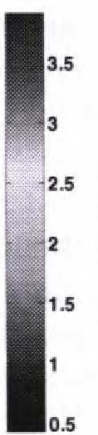

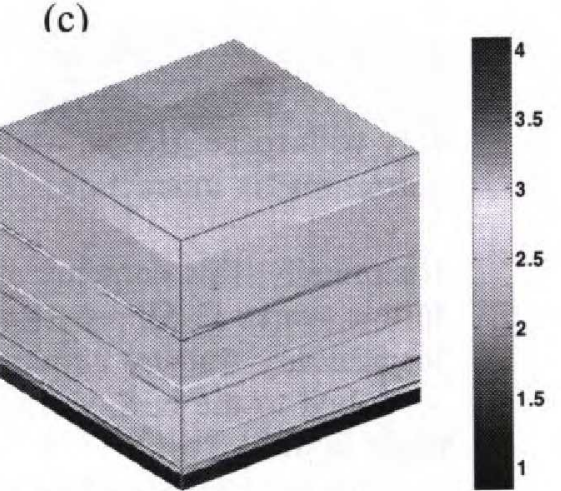

Figure 9: (a) Reference Model 50x50x100, (b) DEGA result 20x20x10, and (c) Geobody result
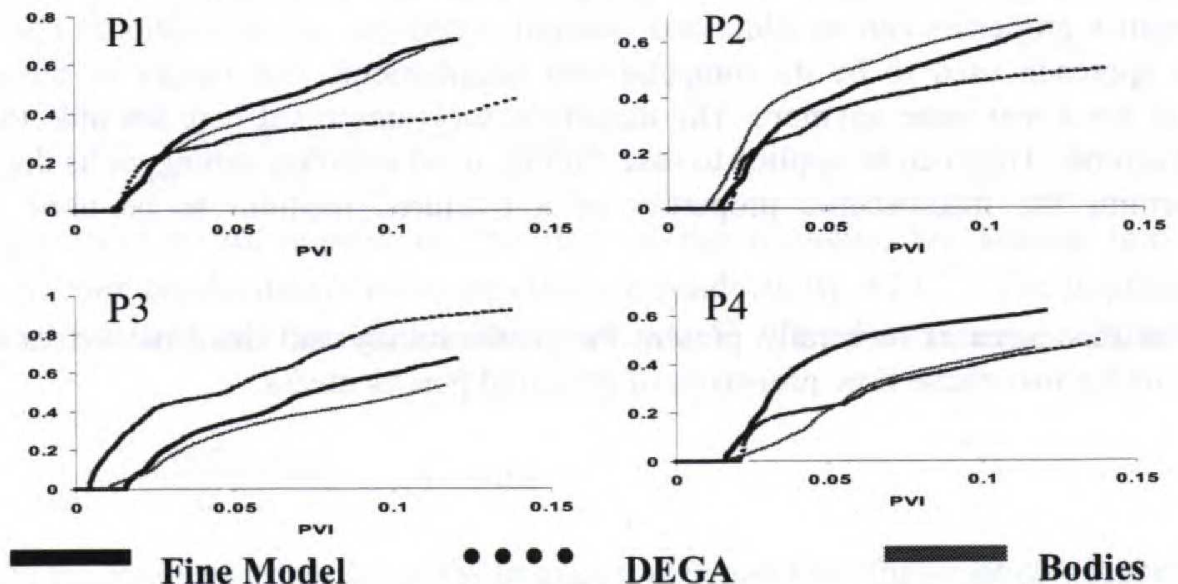

Figure 10: Water cut response results for the four producers. 